\title{
Expression and Function of a Disintegrin and Metalloproteinases in Cancer-Associated Fibroblasts of Colorectal Cancer
}

\author{
Satsuki Mochizuki ${ }^{a}$ Tadakazu Ao $^{a}$ Takumi Sugiura ${ }^{a}$ Keisuke Yonemura ${ }^{a}$ \\ Takehiro Shiraishi $^{a}$ Yoshiki Kajiwara $^{a}$ Koichi Okamoto ${ }^{a}$ Eiji Shinto $^{a}$ \\ Yasunori Okadab Hideki Ueno ${ }^{\mathrm{a}}$ \\ ${ }^{a}$ Department of Surgery, National Defense Medical College, Saitama, Japan; ${ }^{b}$ Department of Pathophysiology for \\ Locomotive and Neoplastic Diseases, Juntendo University Graduate School of Medicine, Tokyo, Japan
}

\section{Keywords}

A disintegrin and metalloproteinase $\cdot$ Cancer-associated fibroblast · Desmoplastic reaction · Colorectal cancer

\begin{abstract}
Background: Cancer tissues consist of cancer cells and stro$\mathrm{ma}$, the latter of which dictates cancer tissue microenvironment. We recently reported that the desmoplastic reaction (DR) pattern at the invasive front in colorectal cancer (CRC) is a promising prognostic indicator. However, the molecular mechanisms of DR formation and contribution to patients' prognosis remain unclear. Summary: The tumor tissue microenvironment composed of extracellular matrix (ECM), soluble factors (growth factors/cytokine/cytokine), and stromal cells controls tumor growth and spread. Among stromal cells, cancer-associated fibroblasts (CAFs) play a key role in development of the cancer tissue microenvironment, and they are responsible for DR formation. CAFs express a disintegrin and metalloproteinases (ADAMs), which modulate cancer tissue microenvironmental factors. We isolated CAFs and normal fibroblasts from colon tissues of patients with CRC and characterized them. CAFs showed the increased expression of several ADAM species including ADAM9,
\end{abstract}

ADAM10, ADAM12, and ADAM17, and the expression was further increased on the ECM-coated plates. Our in vitro and in vivo studies using CAFs and $C R C$ cells suggest that $A D A M$ expression is associated with the morphological DR category, and ADAMs may affect cancer malignancy through tumor proliferation in CRC. Key Message: This review summarizes the present knowledge on ADAMs in cancer and describes our recent findings regarding the molecular biological background of DR mainly by focusing on ADAMs.

(c) 2019 S. Karger AG, Basel

\section{Introduction}

A disintegrin and metalloproteinase (ADAM), ADAM with thrombospondin motifs (ADAMTS), and matrix metalloproteinase (MMP) are gene families belonging to the metzincin superfamily of zinc-based metalloproteinases [1]. The human ADAMTS and MMP gene families are composed of 19 and 23 members, respectively. All of them mainly digest extracellular matrix (ECM) components and non-ECM molecules including growth factors, cytokines, and chemokines $[1,2]$. Apart from them, only 13 of the 21 members of the ADAM gene family exhibit

Satsuki Mochizuki, PhD Department of Surgery National Defense Medical College 3-2 Namiki, Tokorozawa, Saitama 359-8513 (Japan)

E-Mails-mochi@ndmc.ac.jp 
proteolytic activity, and the other 8 members are nonproteolytic ones. Although the substrate specificity of proteolytic ADAMs is narrower than that of ADAMTS and MMP species, their biological functions are diverse, and they are involved in various events such as cell adhesion, fusion, migration, membrane protein shedding, and proteolysis of ECM components $[1,2]$. One of the most important functions of proteolytic ADAM members may be the shedding of membrane proteins, which contributes to the production of soluble active growth factors and cytokines including epidermal growth factor (EGF), heparinbinding EGF-like growth factor (HB-EGF), transforming growth factor alpha (TGF- $\alpha$ ), and tumor necrosis factor- $\alpha$ [1]. They also act as ligands for integrins by competing with matrix proteins [3]. ADAMs are membrane proteins containing a transmembrane domain, but some members such as ADAM9, ADAM12, and ADAM28 have shortsecreted splice variants in addition to their prototype membrane-anchored forms [1]. Thus, they are named as ADAM9m and ADAM9s, ADAM12m and ADAM12s, and ADAM28m and ADAM28s, respectively [1].

Desmoplastic reaction (DR) refers to the overgrowth of fibrous connective tissues around carcinoma cell nests and has recently attracted attention as an indicator of malignant potential $[4,5]$. We previously reported that DR at the invasive front of colorectal cancer (CRC) is a promising prognostic indicator [4] and that the prognostic power of DR categorization in stratifying 5-year relapsefree survival is greater than any other conventional prognostic factors such as TNM stage, venous invasion, and tumor grade [5]. DR in CRC is morphologically classified into the mature, intermediate, and immature types according to the existence of hyalinized keloid-like collagen and myxoid components at the extramural desmoplastic front, and 5 year survival rates of the CRC patients belonging to each type are as follows: mature types (80\%), intermediate types (55\%), and immature types (27\%) [6]. The molecular mechanisms of DR are not well-understood. However, because ECM components in the cancer stroma are produced by fibroblastic cells within cancer tissue, cancer-associated fibroblasts (CAFs) are ascribed to DR in CRC. CAFs are known to modulate the cancer tissue microenvironment by producing ECM molecules, proteolytic enzymes, growth factors, and immunomodulatory cytokines [7-9]. To study the roles of CAFs in the development of DR in CRC, carcinoma cells together with CAFs should be analyzed in a three-dimensional culture system. Therefore, we isolated and characterized CAFs, developed an organoid culture of CRC and a metastasis model, and examined the roles of ADAMs using these systems. In this review article, we review the expression and function of ADAMs derived from cancer cells or CAFs and describe the prospective roles of ADAMs in CRC progression.

\section{Roles of ADAMs in Cancer Cell Proliferation and Progression}

ADAM family members including ADAM9, ADAM10, ADAM12, ADAM15, ADAM17, and ADAM28 are overexpressed in human cancers and are associated with tumor growth and progression [10]. We recently reported overexpression of ADAM9m, but not ADAM9s, in human ovarian clear cell carcinomas and provided evidence that activated ADAM9m expressed on carcinoma cell membrane is involved in cisplatin resistance of clear cell carcinoma cells [11]. Our study suggests that ADAM9m may be an attractive target for therapies for ovarian clear cell carcinomas by combining chemotherapeutics such as cisplatin [11]. In liver cancers, ADAM9 was reported to play an essential role in the shedding of major histocompatibility complex class I-related chain A on hepatocellular carcinoma cells, leading to decreased NK cell activity and evasion of immunity [12]. L1-CAM, a neuronal cell adhesion receptor, and ADAM10 are overexpressed in CRC, and soluble L1-CAM generated by the action of ADAM10 is suggested to contribute to metastasis of CRC cells to the liver [13]. Our previous studies indicated that ADAM28 is overexpressed by carcinoma cells in invasive breast and non-small cell lung carcinomas, showing positive correlations with carcinoma cell proliferation and progression, and that the serum levels of ADAM28 are positively correlated with tumor stage, lymph node metastasis, and carcinoma recurrence in patients with nonsmall cell carcinoma $[14,15]$. ADAM28 degrades von Willebrand factor (VWF), which induces the apoptosis of carcinoma cells by binding to av $\beta 1$ integrin, and thus, ADAM28-expressing carcinoma cells can survive longer in the bloodstream by escaping from VWF-induced apoptosis through digestion of VWF, leading to enhanced metastasis [16]. ADAM28 also promotes angiogenesis by re-activating vascular endothelial growth factor through digestion of connective tissue growth factor in the vascular endothelial growth factor/connective tissue growth factor complex [17]. These data demonstrate that ADAM28 plays an important role in cancer cell proliferation and progression by modulating tumor microenvironmental factors in cancers and suggest that selective inhibitors of ADAM28 may be a therapeutic target for 
these carcinomas. Thus, we recently developed human neutralizing antibodies against ADAM28 and found that one of the antibodies effectively inhibits proliferation and metastasis of lung adenocarcinoma cells in the experimental metastasis mouse models and promotes survival of the carcinoma cell-bearing mice [18]. ADAM9-targeting antibody - drug conjugates for treating cancer have also been developed by a biotechnology company, and they have shown to have effective anticancer effects in mouse models of non-small cell lung cancer, gastric cancer, and CRC [19]. Prospective roles of ADAMs in cancers are summarized in Figure 1a.

\section{ADAMs in Cancer Stroma}

A few studies describe the functions of ADAMs within cancer stroma, and they include ADAM9, ADAM10, ADAM12, and ADAM17. ADAM9s secreted by activated hepatic stellate cells is reported to bind to $\alpha 6 \beta 4$ and $\alpha 2 \beta 1$ integrins of CRC cells and enhance carcinoma cell invasion by digesting laminin in the liver [20]. In melanoma, stromal fibroblast-derived ADAM9 contributes to tumor cell proliferation and apoptosis by increasing the expression of tissue inhibitor of metalloproteinases- 1 and soluble TNF-receptor 1, respectively [21]. ADAM12 is highly expressed by stromal cells in mouse prostate tumors and plays a critical role in tumor progression [22]. This study suggested the potential mechanism in which HB-EGF, EGF, and/or beta-cellulin released by the action of ADAM12 expressed on tumor stromal cells activate tumor cell proliferation in a paracrine manner [22]. Most recently, ADAM12 was reported to be a specific marker of stromal CAFs in esophageal adenocarcinoma, and the patients with high serum levels of ADAM12 showed a poor response to chemoradiation therapy [23]. Rhomboid 5 homolog 2 promotes cleavage of TGF $\beta$ receptor 1 by activating ADAM17 and motility of CAFs in response

Fig. 1. Prospective roles of ADAMs in cancers and expression of ADAMs by CAFs from CRC tissue. a Implications of ADAMs for various biological events in cancers through interaction and/or digestion of tissue microenvironmental factors. ADAMs bind to integrins, ECM proteins, and process growth factors, cytokines, chemokines, or cell surface proteins including growth factor receptors. These processes modulate various biological events in cancers. b CRC tissues were cut into $2-3-\mathrm{mm}$ fragments and placed in culture medium for 2 weeks to allow for migration and proliferation of CAFs. c CAFs were cultured on noncoated and ECM (Matrigel)coated plates. Note that CAFs exhibit aggregated coalescent mor- to TGF $\beta$ [24]. The study suggested that inhibition of ADAM17 activity in CAFs blocks the development CAF properties and thereby inhibits gastric cancer cell invasion [24]. ADAM17-mediated TGF- $\alpha$ released from stromal fibroblasts within breast carcinomas contributes to tumor cell proliferation and progression [25]. Fibroblasts lacking all 4 members of the tissue inhibitor of metalloproteinases family are known to exhibit molecular features of CAFs, generate exosomes with enhanced MMP expression and ADAM10 activity, and associate with enhanced cancer cell motility, metabolic reprogramming, and induction of cancer stem cell features [26]. These data indicate that the CAF-derived ADAMs are implicated in modulation of cancer tissue microenvironmental factors.

\section{Approaches to Functional Analyses of CAF-Derived ADAMs in CRC}

There have been no systematic studies about ADAMs in CAFs in CRC. Therefore, we isolated primary CAFs and normal fibroblasts (NFs) from the surgically resected colon tissues of the patients with CRC. CAFs were obtained by the explant culture of the CRC tissue. They started to migrate from the CRC tissue fragments at day 1 , growing around them at days $2-4$, and the CRC tissue fragments were surrounded by adherent CAFs at days 10-12 (Fig. 1b). The tissue fragments were removed from the CAFs at days 10-14. Isolated CAFs were found to be positive for the specific CAF markers including $\alpha$-smooth muscle actin, fibroblast-specific protein-1, and fibroblast activation protein by reverse RT-PCR and immunoblotting. CAFs were then passed twice and used for our experiments at passages from 3 to 7 . CAFs showed a monotonous spindle-shaped structure on plastic plates, but they occasionally exhibited aggregated coalescent morphology on plates coated with Matrigel, containing laminin, type IV collagen, nidogen, and perlecan (Fig. 1c).

phology on ECM-coated plates. d Expression of ADAM9 and ADAM17 in CAFs of CRC by immunoblotting. e ADAM9 expression is elevated under culture of CAFs on ECM-coated plates. ADAMs, a disintegrin and metalloproteinases; VWF, von Willebrand factor; HB-EGF, heparin-binding epidermal growth factor; TGF, transforming growth factor; CAF, cancer-associated fibroblasts; VEGF, vascular endothelial growth factor; CTGF, connective tissue growth factor; ECM, extracellular matrix; CXCL12, C$\mathrm{X}-\mathrm{C}$ motif chemokine 12; IL, interleukin; IGF, insulin-like growth factor; IGFBP-3, IGF binding protein 3; TNFR1, tumor necrosis factor receptor 1 .

(For figure see next page.)
Mochizuki et al. 

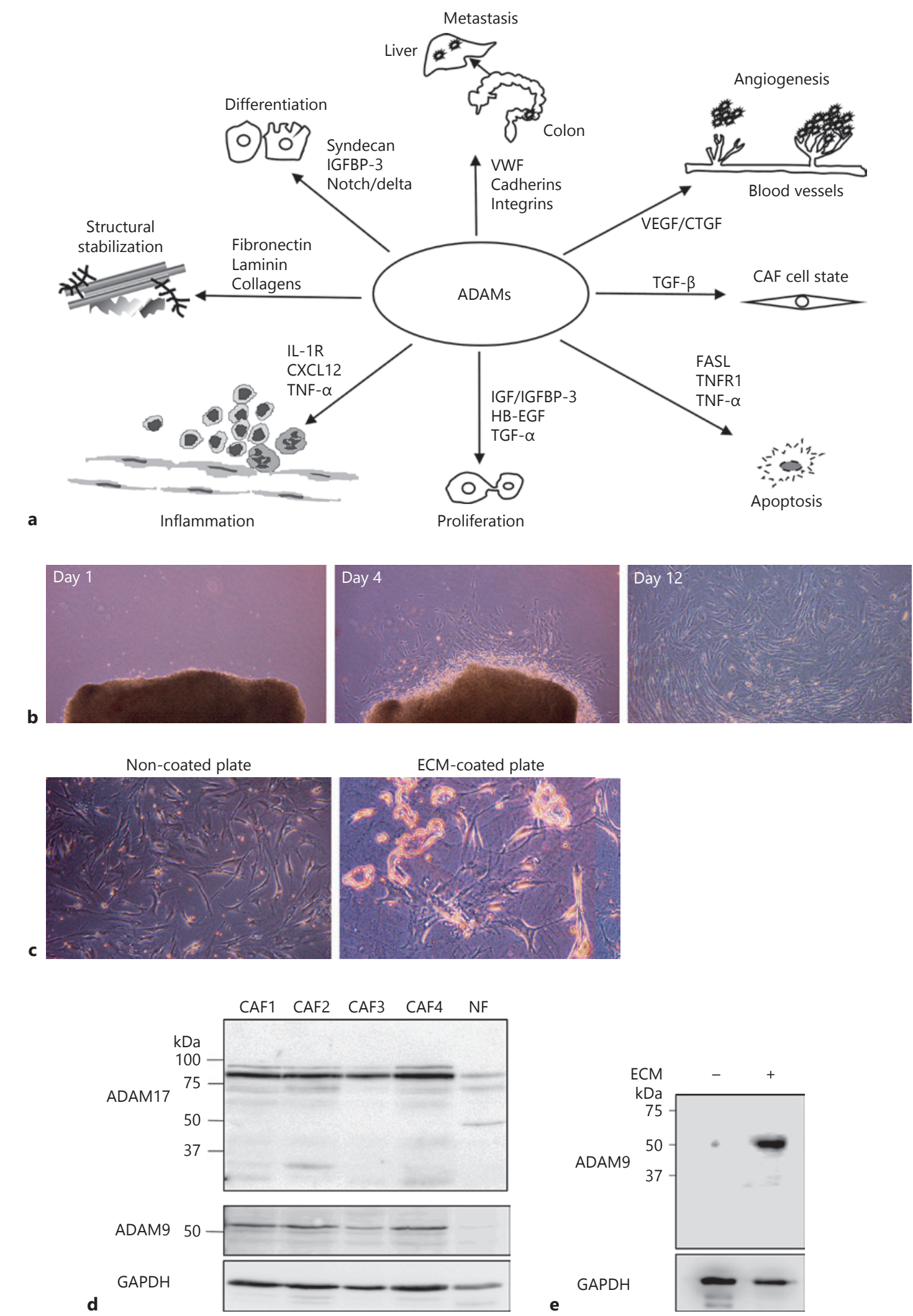

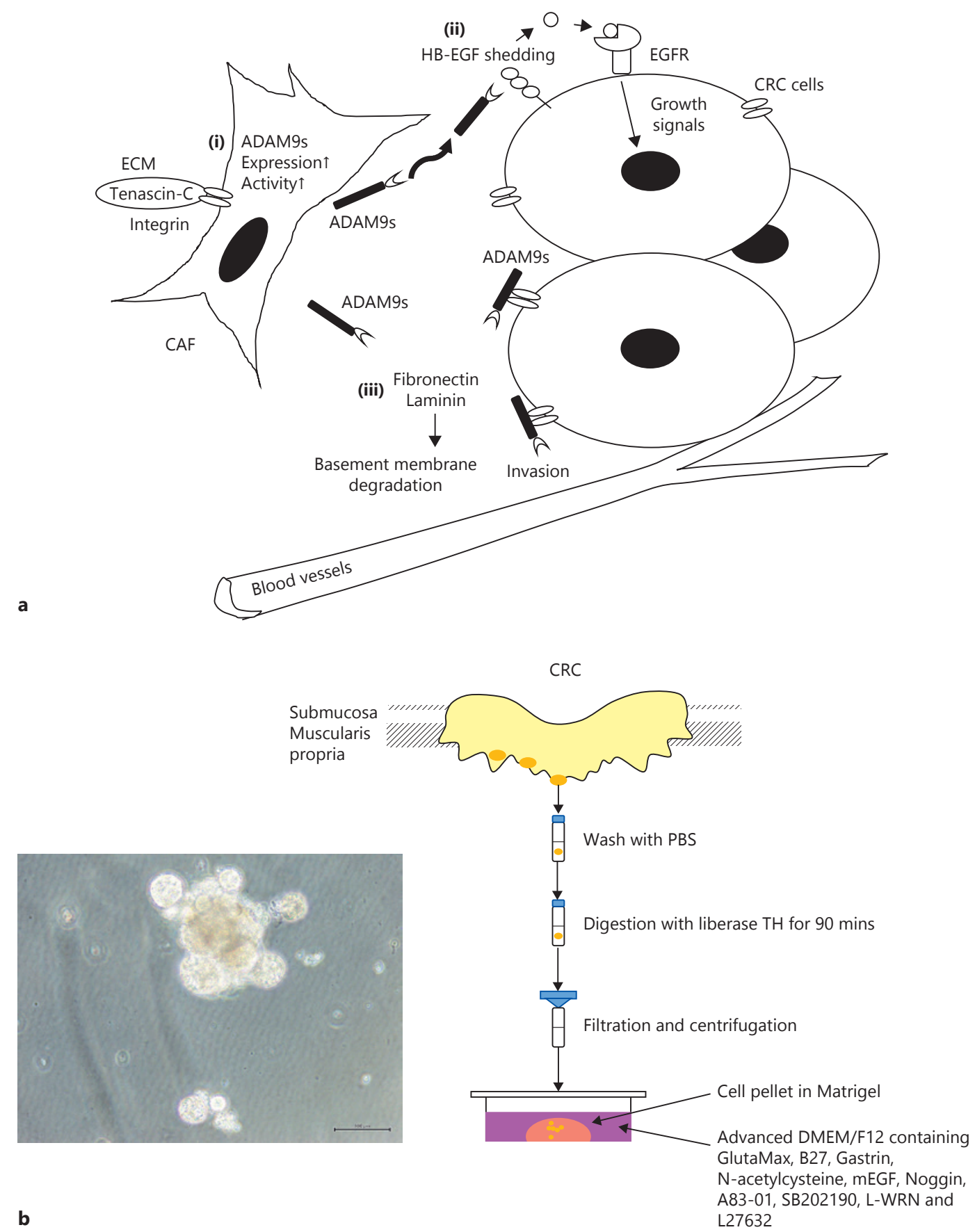

Fig. 2. Prospective roles of ADAM9s in CRC progression and organoid culture from CRC. a Tenascin-C or ECM molecules induces expression and proteolytic activity of ADAM9s by binding to integrins (i). ADAM9s secreted by CAFs induces HB-EGF shedding on CRC cells, and then enhances the proliferation of CRC cells through EGF receptor signaling pathway (ii). ADAM9s degrades basement membrane ECM components such as laminin and fibronectin to promote migration and invasion (iii). b Scheme of organoid culture methods. Identification of living human CRCderived organoid, showing tightly packed cell nests with clearly defined edges. The scale bar represents $100 \mu \mathrm{m}$. ADAMs, a disintegrin and metalloproteinases; HB-EGF, heparin-binding epidermal growth factor; ECM, extracellular matrix; CRC, colorectal cancer; CAF, cancer-associated fibroblast; EGFR, estimated glomerular filtration rate. 
When the expression of ADAMs and MMPs was examined by RT-PCR, several ADAMs (ADAM9, ADAM10, ADAM12, and ADAM17) and MMPs (MMP1, MMP2, MMP3, and MT1-MMP) were expressed in CAFs, but not in NFs (manuscript in preparation). Importantly, immunoblotting analysis indicated that ADAM9s and ADAM17 in CAFs are expressed in the $55-\mathrm{kDa}$ active form of ADAM9s and the $80-\mathrm{kDa}$ active form of ADAM17, whereas only negligible expression of these ADAMs was detected in NFs (Fig. 1d). In addition, the ADAM9s expression was promoted by culturing CAFs on the Matrigel-coated plates compared with the culture on the noncoated plates (Fig. 1e). Given our observation that CAFs display higher expression of active ADAM9s form on the ECM culture, ECM molecules may induce outside-in cell signaling through binding to integrins. In fact, tenascin- $\mathrm{C}$ is known to increase ADAM9s proteolytic activity to the ECM components and promote migration and invasion through integrins [27]. We have observed that tenascin- $\mathrm{C}$ and fibronectin are highly expressed in the immature stroma of CRC, and both molecules are known to be related to CRC aggressiveness and decreased survival time of the patients in various malignancies [4]. In our preliminary experiments, we obtained the data that the conditioned media of CAFs isolated from the CRC tissues with the immature DR have stimulative effects on CRC cell proliferation, and xenografts of CRC cells together with these CAFs rapidly grow (manuscript in preparation). ADAM9s is reported to cleave amyloid precursor protein, pro-HB-EGF, collagen XVII, p75 neurotrophin receptor, fibronectin, laminin, fibroblast growth factor receptor 2, and gelatin, digestion of which is implicated in various pathologies including cancer $[1,2]$. Taken together, these data suggest that ADAM9s activity upregulated by the interaction of tenascin- $\mathrm{C}$ with integrin on CAFs is involved in CRC cell proliferation and progression through several pathways such as the estimated glomerular filtration rate ligand shedding/estimated glomerular filtration rate activation axis and digestion of basement membrane components such as laminin and fibronectin (Fig. 2a). However, further studies are definitely needed to explore the molecular mechanisms.

To examine underlying mechanisms of CRC metastasis, we established an experimental mouse model for monitoring cancer cell metastasis by in vivo bioluminescence imaging according to our previous methods [16]. In this method, transplanted CRC cells (HT-29) expressing Luciferase and Venus (a variant of green fluorescent protein), named as HT- $29^{\text {ffluc-cp } 156}$ cells, can be readily de- tected within the tissues of live animals after luciferin administration [16]. Thus, tumor development was monitored over time using an in vivo imaging system. Analyses of the role of ADAMs in metastasis of CRC cells are currently underway.

The organoid culture method for colonic epithelium was developed by Sato et al. [28] by using Matrigel as a three-dimensional matrix, in which either intestinal crypts or Lgr5+ intestinal epithelium cells were embedded. This method was optimized to produce organoids from mouse and human colon and colorectal adenoma tissues as well as those from human colonic stem cells $[29,30]$. This organoid culture system would be an effective method for conducting biological and pathological analysis of DR because it is a more functionally relevant model to the in vivo response compared to traditional 2D culture and provides the effective missing link between in vitro and in vivo studies. Using the organoid culture methods, we established a human CRCderived organoid culture system (Fig. 2b) and are now studying the functions of CAFs in organoids in our laboratory.

\section{Conclusions}

Our data demonstrate that the expression of ADAMs is associated with the morphological DR category and suggest that ADAMs affect cancer malignancy by modulating tumor microenvironmental factors in CRC. The disruption of ADAMs activity using ADAM-targeting antibodies may block the development of CAFs and thereby inhibit invasive cancer growth. However, further studies are necessary to fully understand the role of ADAMs in CRC cell proliferation and progression.

\section{Acknowledgments}

We thank Dr. Masanobu Ohshima and Dr. Mizuho Nakayama (Cancer Research Institute, Kanazawa University) for their kind suggestions about organoid culture, and all members of the Dr. Ohshima laboratory for their support and discussions. This work was supported by JSPS KAKENHI Grant Number 18K07052 and External Collaborative Research Grant of Cancer Research Institute, Kanazawa University to Satsuki Mochizuki.

\section{Disclosure Statement}

The authors have no conflicts of interest to declare. 


\section{References}

1 Edwards DR, Handsley MM, Pennington CJ. The ADAM metalloproteinases. Mol Aspects Med. 2008 Oct;29(5):258-89.

2 Murphy G. Regulation of the proteolytic disintegrin metalloproteinases, the 'Sheddases'. Semin Cell Dev Biol. 2009 Apr;20(2):138-45.

3 Seals DF, Courtneidge SA. The ADAMs family of metalloproteases: multidomain proteins with multiple functions. Genes Dev. 2003 Jan; 17(1):7-30.

4 Ueno H, Shinto E, Shimazaki H, Kajiwara Y, Sueyama T, Yamamoto J, et al. Histologic categorization of desmoplastic reaction: its relevance to the colorectal cancer microenvironment and prognosis. Ann Surg Oncol. 2015 May;22(5):1504-12.

5 Ueno H, Kanemitsu Y, Sekine S, Ishiguro M, Ito E, Hashiguchi Y, et al. Desmoplastic pattern at the tumor front defines poor-prognosis subtypes of colorectal cancer. Am J Surg Pathol. 2017 Nov;41(11):1506-12.

6 Ueno H, Jones AM, Wilkinson KH, Jass JR, Talbot IC. Histological categorisation of fibrotic cancer stroma in advanced rectal cancer. Gut. 2004 Apr;53(4):581-6.

7 Koliaraki V, Pallangyo CK, Greten FR, Kollias G. Mesenchymal Cells in Colon Cancer. Gastroenterology. 2017 Apr;152(5):964-79.

8 Kuzet SE, Gaggioli C. Fibroblast activation in cancer: when seed fertilizes soil. Cell Tissue Res. 2016 Sep;365(3):607-19.

9 Rizvi S, Gores GJ. Current diagnostic and management options in perihilar cholangiocarcinoma. Digestion. 2014;89(3):216-24.

10 Mochizuki S, Okada Y. ADAMs in cancer cell proliferation and progression. Cancer Sci. 2007 May;98(5):621-8.

11 Ueno M, Shiomi T, Mochizuki S, Chijiiwa M, Shimoda M, Kanai Y, et al. ADAM9 is over-expressed in human ovarian clear cell carcinomas and suppresses cisplatin-induced cell death. Cancer Sci. 2018 Feb; 109(2):471-82.

12 Kohga K, Takehara T, Tatsumi T, Ishida H, Miyagi T, Hosui A, et al. Sorafenib inhibits the shedding of major histocompatibility complex class I-related chain A on hepatocellular carcinoma cells by down-regulating a disintegrin and metalloproteinase 9. Hepatology. 2010 Apr;51(4):1264-73.
13 Gavert N, Sheffer M, Raveh S, Spaderna S, Shtutman M, Brabletz T, et al. Expression of L1-CAM and ADAM10 in human colon cancer cells induces metastasis. Cancer Res. 2007 Aug;67(16):7703-12.

14 Mitsui Y, Mochizuki S, Kodama T, Shimoda M, Ohtsuka T, Shiomi T, et al. ADAM28 is overexpressed in human breast carcinomas: implications for carcinoma cell proliferation through cleavage of insulin-like growth factor binding protein-3. Cancer Res. 2006 Oct; 66(20):9913-20.

15 Kuroda H, Mochizuki S, Shimoda M, Chijiiwa M, Kamiya K, Izumi Y, et al. ADAM28 is a serological and histochemical marker for non-small-cell lung cancers. Int J Cancer. 2010 Oct;127(8):1844-56.

16 Mochizuki S, Soejima K, Shimoda M, Abe H, Sasaki A, Okano HJ, et al. Effect of ADAM28 on carcinoma cell metastasis by cleavage of von Willebrand factor. J Natl Cancer Inst. 2012 Jun;104(12):906-22.

17 Mochizuki S, Tanaka R, Shimoda M, Onuma J, Fujii Y, Jinno H, et al. Connective tissue growth factor is a substrate of ADAM28. Biochem Biophys Res Commun. 2010 Nov;402(4):651-7.

18 Mochizuki S, Shimoda M, Abe H, Miyamae Y, Kuramoto J, Aramaki-Hattori N, et al. Selective inhibition of ADAM28 suppresses lung carcinoma cell growth and metastasis. Mol Cancer Ther. 2018 Nov;17(11):2427-38.

19 Hicks SW, Yoder NC, Loo D, Muvaffak A, Zhou Y, Fuller ME, et al. Abstract 37: Novel antibody-drug conjugates targeting $\mathrm{AD}$ AM9-expressing solid tumors demonstrate potent preclinical activity. Cancer Res. DOI: 10.1158/1538-7445.

20 Mazzocca A, Coppari R, De Franco R, Cho JY, Libermann TA, Pinzani M, et al. A secreted form of ADAM9 promotes carcinoma invasion through tumor-stromal interactions. Cancer Res. 2005 Jun;65(11):4728-38.

21 Abety AN, Fox JW, Schönefuß A, Zamek J, Landsberg J, Krieg T, et al. Stromal fibroblastspecific expression of ADAM-9 modulates proliferation and apoptosis in melanoma cells in vitro and in vivo. J Invest Dermatol. 2012 Oct;132(10):2451-8.

22 Peduto L, Reuter VE, Sehara-Fujisawa A Shaffer DR, Scher HI, Blobel CP. ADAM12 is highly expressed in carcinoma-associated stroma and is required for mouse prostate tumor progression. Oncogene. 2006 Aug; 25(39):5462-6.

23 Ebbing EA, van der Zalm AP, Steins A, Creemers A, Hermsen S, Rentenaar R, et al. Stromal-derived interleukin 6 drives epithelial-to-mesenchymal transition and therapy resistance in esophageal adenocarcinoma. Proc Natl Acad Sci USA. 2019 Feb;116(6): 2237-42.

24 Ishimoto T, Miyake K, Nandi T, Yashiro M, Onishi N, Huang KK, et al. Activation of transforming growth factor beta 1 signaling in gastric cancer-associated fibroblasts increases their motility, via expression of rhomboid 5 homolog 2, and ability to induce invasiveness of gastric cancer cells. Gastroenterology. 2017 Jul;153(1):191-204.e16.

25 Gao MQ, Kim BG, Kang S, Choi YP, Yoon JH, Cho NH. Human breast cancer-associated fibroblasts enhance cancer cell proliferation through increased TGF- $\alpha$ cleavage by ADAM17. Cancer Lett. 2013 Aug;336(1): 240-6.

26 Shimoda M, Principe S, Jackson HW, Luga V, Fang H, Molyneux SD, et al. Loss of the Timp gene family is sufficient for the acquisition of the CAF-like cell state. Nat Cell Biol. 2014 Sep;16(9):889-901.

27 Brösicke N, van Landeghem FK, Scheffler B, Faissner A. Tenascin-C is expressed by human glioma in vivo and shows a strong association with tumor blood vessels. Cell Tissue Res. 2013 Nov;354(2):409-30.

28 Sato T, Vries RG, Snippert HJ, van de Wetering M, Barker N, Stange DE, et al. Single Lgr5 stem cells build crypt-villus structures in vitro without a mesenchymal niche. Nature. 2009 May;459(7244):262-5.

29 Sato T, Stange DE, Ferrante M, Vries RG, Van Es JH, Van den Brink S, et al. Long-term expansion of epithelial organoids from human colon, adenoma, adenocarcinoma, and Barrett's epithelium. Gastroenterology. 2011 Nov; 141(5): 1762-72.

30 Jung P, Sato T, Merlos-Suárez A, Barriga FM, Iglesias $\mathrm{M}$, Rossell $\mathrm{D}$, et al. Isolation and in vitro expansion of human colonic stem cells. Nat Med. 2011 Sep;17(10):1225-7. 